University of Wollongong

Research Online

Faculty of Informatics - Papers (Archive)

Faculty of Engineering and Information

Sciences

April 2003

\title{
Blind deconvolution of dynamical systems using a balanced parameterized state space approach
}

Ah Chung Tsoi

University of Wollongong, act@uow.edu.au

L. S. Ma

University of Wollongong

Follow this and additional works at: https://ro.uow.edu.au/infopapers

Part of the Physical Sciences and Mathematics Commons

\section{Recommended Citation}

Tsoi, Ah Chung and Ma, L. S.: Blind deconvolution of dynamical systems using a balanced parameterized state space approach 2003.

https://ro.uow.edu.au/infopapers/101

Research Online is the open access institutional repository for the University of Wollongong. For further information contact the UOW Library: research-pubs@uow.edu.au 


\title{
Blind deconvolution of dynamical systems using a balanced parameterized state space approach
}

\author{
Abstract \\ In this paper, the problem of blind deconvolution of dynamical systems is considered using a state space \\ approach. A balanced parameterized canonical form is used as a model for the underlying dynamical \\ system instead of the more common controller or observable canonical form. The results are compared \\ with those obtained using a controller canonical form. It is shown experimentally that using the balanced \\ parameterized canonical form is more robust than the ones using a controller canonical form.

\section{Keywords} \\ blind source separation deconvolution, parameter estimation, state-space methods

\section{Disciplines} \\ Physical Sciences and Mathematics

\section{Publication Details} \\ This paper originally appeared as: Tsoi, AC \& Ma, LS, Blind deconvolution of dynamical systems using a \\ balanced parameterized state space approach, Proceedings, IEEE International Conference on Acoustics, \\ Speech, and Signal Processing, 6-10 April 2003, 4, 309-312. Copyright IEEE 2003.
}




\title{
BLIND DECONVOLUTION OF DYNAMICAL SYSTEMS USING A BALANCED PARAMETERIZED STATE SPACE APPROACH
}

\author{
A C Tsoi, LSMa \\ University of Wollongong, Northfields Avenue \\ Wollongong, NSW 2522, Australia
}

\begin{abstract}
In this paper, the problem of blind deconvolution of dynamical systems is considered using a state space approach. A balanced parameterized canonical form is used as a model for the underlying dynamical system instead of the more common controller or observable canonical form. The results are compared with those obtained using a controller canonical form. It is shown experimentally that using the balanced parameterized canonical form is more robust than the ones using a controller canonical form.
\end{abstract}

\section{INTRODUCTION}

Blind deconvolution or separation of source signals which had been mixed by a dynamical system has been extensively studied in recent years $[1,2,8]$. In this paper we wish to study the blind deconvolution of source signals of dynamical systems of the following form:

$$
\begin{aligned}
\mathbf{x}(k+1) & =A \mathbf{x}(k)+B \mathbf{s}(k) \\
\mathbf{u}(k) & =C \mathbf{x}(k)+D \mathbf{s}(k)
\end{aligned}
$$

where $\mathbf{s}(k) \in \mathcal{R}^{n}$ is the source signal. $\mathbf{u}(k) \in \mathcal{R}^{n}$ is the sensor output of this linear time invariant (LTI) system. $\mathrm{x}(k) \in \mathcal{R}^{N}$ is the state of the dynamical system!

The problem can be stated as follows: given the sensor output $\mathbf{u}(k)$, is it possible to recover the source signals $\mathbf{s}(k)$. In general, this is not possible. However, if we place a number of assumptions on the source signals then it becomes possible to recover the source signals from the sensor outputs. The usual assumptions placed on the source signals are as follows [2]:

\section{The source signals are independent.}

Thanks to the Australian Research Council, and Motorola Australia Research Centre for partial funding of this research.

${ }^{1}$ Note that we assume that there are as many sensors as there are number of sources. This is a particular case of the more general case when there may be an unequal number of sensors and source signals. In this paper, we will not deal with this more general case, but instead concentrate on the particular case when there are equal number of sensors and source signals.
2. At most one of the source signals is a Gaussian distributed signal.

3. It is only possible to recover the source signals modulo scale invariance, and polarity.

Since we assume that the source signals are mixed by a dynamical system, we need to place some further assumptions on the dynamical system itself, Common assumptions on the dynamical systems [6] are as follows:

1. The dynamical system must be causal.

2. The dynamical system is assumed to be time invariant.

3. The dynamical system is assumed to linear.

This problem set in a state space formulation has been studied by Zhang and Cichocki [8]. To reduce the number of parameters to be estimated, they have assumed a controller canonical form for the parameters $A, B, C, D$. This is a valid assumption as it is known that a linear time invariant (LTI) dynamical system is invariant under coordinate transformations. Hence, the general LTI system as expressed in $\mathrm{Eq}(1)$ and $\mathrm{Eq}(2)$ can be transformed into equivalent representations, e.g., the controller canonical form using coordinate transformations.

However, there are a number of issues associated with the approach taken in [8].

- They require the dimension $N$ to be estimated. They have indicated that such value can be estimated using, e.g., Akaike Information Criterion or the Final Prediction Error criterion. They have further indicated that if the dimension is over estimated then it will introduce additional delays in the recovered signals.

- From system theory, it is known that the controller canonical form has some difficulties, in particular, when it is used as a parameterization in system identification studies. These difficulties include the possibility of ill conditioning, i.e., the parameter estimation process may become unstable due to pole-zero cancellation. 
It is also known in system theory the difficulties encountered in the application of controller canonical form can be overcome to a large extent by the use of balanced parameterization, which is another possible parameterization of linear systems [3, 4]. In this case, we need to assume that the LTI system is stable. This is a reasonable assumption in most practical systems.

In this paper, our aim is to explore the possibility of using balanced parameterization of the LTI system in blind deconvolution of source signals. While we have not resolved completely in this paper the difficulties encountered in the requirement to estimate the dimension $N$, the proposed method at least overcomes some of the difficulties encountered in the implementation of the approach used in [8], e.g., instability of the parameter estimation process.

The structure of the paper is as follows: in Section 2, we will give a brief introduction to the balanced realization parameterization of linear systems. In Section 3 we will give a description of the estimation algorithm of the unknown parameters in the balanced parameterized model, while in Section 4 we will give some comparative results using the proposed technique and the technique developed by [8]. It is shown experimentally that using balanced parameterization, the results appear to be more robust. Some conclusions are drawn in Section 5.

\section{BALANCED PARAMETERIZATION OF LINEAR TIME INVARIANT SYSTEMS}

Consider a discrete linear time invariant system ${ }^{2}$ :

$$
\begin{aligned}
\mathbf{x}(k+1) & =A_{d} \mathbf{x}(k)+B_{d} \mathbf{u}(k) \\
\mathbf{y}(k) & =C_{d} \mathbf{x}(k)+D_{d} \mathbf{u}(k)
\end{aligned}
$$

where $\mathbf{u} \in \mathcal{R}^{n}$ and $\mathbf{y} \in \mathcal{R}^{n}$, and $\mathbf{x} \in \mathcal{R}^{N}$. If the system is asymptotically stable, the controllability Grammian $P_{d}$ and the observability Grammian $Q_{d}$, both $N \times N$ matrices, are respectively given by the following dual discrete time Lyapunov equations:

$$
\begin{aligned}
P_{d}-A_{d} P_{d} A_{d}^{T} & =B_{d} B_{d}^{T} \\
Q_{d}-A_{d}^{T} Q_{d} A_{d} & =C_{d}^{T} C_{d}
\end{aligned}
$$

Discrete time balanced realization is defined when $P_{d}=$ $Q_{d}=\Sigma_{d}=\operatorname{diag}\left(\sigma_{1}, \sigma_{2}, \ldots, \sigma_{N}\right)$. The quantities $\sigma_{i}, i=$ $1,2, \ldots, N$ are called the Hankel singular values.

In a similar fashion, it is possible to define balanced retaliation of continuous LTI systems. In this case, consider:

\footnotetext{
${ }^{2}$ Note that in this paper we will not give the most general formulation of balanced realization or parameterization, as this can be found elsewhere in the literature; see e.g., [7]. We will only give the formulation which has the same number of inputs and outputs, which is the case considered here.
}

$$
\begin{aligned}
\dot{\mathbf{x}} & =A_{c} \mathbf{x}+B_{c} \mathbf{u} \\
\mathbf{y} & =C_{c} \mathbf{x}+D_{c} \mathbf{u}
\end{aligned}
$$

where $\mathbf{y} \in \mathcal{R}^{n}, \mathbf{u} \in \mathcal{R}^{n}$, and $\mathbf{x} \in \mathcal{R}^{N}$. The controllability Grammian $P_{c}$ and observability Grammian $Q_{c}$, both $N \times N$ matrices, are respectively given by the solution to the dual Lyapunov equations as follows:

$$
\begin{aligned}
A_{c} P_{c}+P_{c} A_{c}^{T} & =-B_{c} B_{c}^{T} \\
A_{c}^{T} Q_{c}+Q_{c} A_{c} & =-C_{c}^{T} C_{c}
\end{aligned}
$$

The system is said to be balanced if $P_{c}=Q_{c}=\Sigma_{c}=$ $\operatorname{diag}\left(\sigma_{1}, \sigma_{2}, \ldots, \sigma_{N}\right)$.

Note that in both the continuous time case and in the discrete time case, the balanced realization is defined through the diagonalisation of the controllability Grammian and the observability Grammian. If the system $A_{c}, B_{c}, C_{c}, D_{c}$ or $A_{d}, B_{d}, C_{d}, D_{d}$ are given, this can be performed quite easily. However, if the system is not known and needs to be estimated from input output data, then it is quite difficult to obtain the unknown system, as well as satisfying the dual Lyapunov equations with a diagonal matrix. As a result, a number of researchers proposed balanced parameterization models $[7,3,4]$. These are parameterized models which will yield a balanced realized state space model, i.e., it allows us to find the particular set of parameters from input output data which will give a diagonal matrix solution to the dual Lyapunov equations.

Consider a linear continuous time invariant system with $N$ states, and $n$ inputs and $n$ outputs, parameterized by the set of parameters $\Theta=\left\{\Sigma, B_{c}, D_{c}, \phi\right\}$, where

$\Sigma \quad$ is the set of singular values, given by $\sigma_{1}>\sigma_{2}$ $>. .>\sigma_{N}, \sigma_{N}>0$.

$\phi \quad \phi=\left\{\begin{array}{llll}\phi_{1} & \phi_{2} & \ldots & \phi_{N}\end{array}\right\}$.

$\phi_{j}$ is a $n-1$ vector and its elements $\phi_{j i} \in$ $\left[-\frac{\pi}{2}, \frac{\pi}{2}\right]$.

$B_{c} \quad B_{c}=\left\{\begin{array}{llll}B_{c}(1) & B_{c}(2) & \ldots & B_{c}(N)\end{array}\right\}$ $B_{\mathrm{c}}(j)$ is $n$ row vector with real values, the first element of the row is positive

$D_{c} \quad n \times n$ real matrix

Note that here we have taken a simplifying assumption: all the Hankel singular values are distinct. This assumption simplifies a number of the structural parameters, which are usually associated with multiple Hankel singular values. In practice, this assumption is usually satisfied, as there are always numerical errors which prevent one Hankel singular value to be exactly the same as another Hankel singular value.

The state space matrices $A_{c}, B_{c}, C_{c}, D_{c}$ can be obtained from this parameter set as follows: 


$$
\begin{aligned}
& B_{c} \quad B_{c}^{T}=\left[\begin{array}{llll}
B_{c}(1)^{T} & B_{c}(2)^{T} & \ldots & B_{c}(N)^{T}
\end{array}\right] \\
& C_{c} \quad C_{c}=\left[\begin{array}{llll}
C_{c}(1) & C_{c}(2) & \ldots & C_{c}(N)
\end{array}\right] \\
& \text { where } C_{c}(j)=U(j) \sqrt{B_{c}(j) B_{c}(j)^{T}} \\
& U\left(\phi_{j}\right) \text { is an } n \text { vector } \\
& A_{c} \quad A_{c}=\left[a_{i j}\right] \text { for } i, j=1,2, \ldots, N \\
& D_{c} \quad D_{c} \text { is an } n \times n \text { matrix }
\end{aligned}
$$

The elements of $U$ are given as follows:

$$
U(j)=\left[\begin{array}{llll}
u_{1 j} & u_{2 j} & \ldots & u_{n j}
\end{array}\right]
$$

where the elements of $u_{i}$ are given as follows:

$$
\begin{aligned}
u_{1 j} & =\cos \phi_{n-1, j} \cos \phi_{n-2, j} \ldots \cos \phi_{2, j} \cos \phi_{1}(12) \\
u_{2 j} & =\cos \phi_{n-1, j} \cos \phi_{n-2, j} \ldots \cos \phi_{2, j} \sin \phi_{1}(13) \\
u_{3 j} & =\cos \phi_{n-1, j} \cos \phi_{n-2, j} \ldots \sin \phi_{2, j} \\
u_{4 j} & =\cos \phi_{n-1, j} \cos \phi_{n-2, j} \ldots \sin \phi_{3, j} \\
\vdots & \\
u_{(n-1) j} & =\cos \phi_{n-1, j} \sin \phi_{n-2, j} \\
u_{n j} & =\sin \phi_{n-1, j}
\end{aligned}
$$

The elements $a_{i j}$ of the matrix $A_{c}$ is given by the following:

$a_{i j}=\left\{\begin{array}{lr}-\frac{1}{2 \sigma_{j}} B_{c}(j) B_{c}(j)^{T} & \mathrm{i}=\mathrm{j} \\ \frac{1}{\left(\sigma_{i}^{2}-\sigma_{j}^{2}\right)}\left(\sigma_{j} B_{c}(j) B_{c}(j)^{T}-\sigma_{i} C_{c}(i)^{T} C_{c}(j)\right) & \mathrm{i} \neq \mathrm{j}\end{array}\right.$

The matrices $A_{c}, B_{c}, C_{c}, D_{c}$ is balanced with Grammian $\Sigma=\operatorname{diag}\left(\sigma_{1}, \sigma_{2}, \ldots, \sigma_{N}\right)$.

This is the parameterization of the continuous LTI system. There are a total of $N+N(n-1)+N n+n^{2}=$ $(2 N+n) n$ parameters. Note that the number of unknown parameters is more than the corresponding ones in the controller canonical form. Intuitively speaking, it is these extra parameters which allow the parameter estimation algorithm to perform better than the parameterization using controller canonical form.

\section{DESCRIPTION OF PARAMETER ESTIMATION ALGORITHM}

It is noted that the balanced parameterization is given only in terms of a continuous LTI system. However, the measurements of sensor outputs are assumed to be in discrete time, as a result, we will need to convert the continuous time model using a bilinear transformation into a corresponding discrete LTI model. This will permit us to use the measurement data to estimate the parameters of the corresponding discrete LTI model.

The parameters of the discrete LTI system can be estimated using the general parameter estimation algorithms provided by [8].
Now once the parameters are updated using the algorithms given in [8], we will need to perform the inverse bilinear transformation into a corresponding continuous LTI system setting. Through the parameter relationship between the continuous LTI system parameters and the balanced parameterization, it is possible to work out a set of new parameter values for the balanced parameterization which can be used to update them. Once the parameters are updated, the cycle can begin again.

The question one wish to ask is: why do we need to go through this circuitous route in order to obtain an updating algorithm for the parameters of the balanced parameterization form. The answer to this question lies in two aspects:

1. The development of the theory on system identification using balanced parameterization is only available in the continuous time setting. The corresponding theory of system identification on discrete time balanced parameterization is still unknown. This is the reason why we need to perform the bilinear transformation so that we can make use of the data which is assumed to be available in discrete time. Obviously if we assume that the sampling interval is short then we can use the continuous time model directly. However, in our studies we do not make this assumption. Hence we need to develop a general theory which will allow us to make use of the discrete time data.

2. Had we tackle the parameter estimation of the balanced realization directly, we will need to satisfy the dual Lyapunov equations at every step. Developing such an algorithm is a challenge, as it is not immediately clear how this can be performed.

A general structure of the parameter estimation can be described as follows:

Step 1 From the set of parameters $\Theta$, obtain the continuous time balanced parameterization.

Step 2 Use bilinear transformation to transform this continuous LTI system into the corresponding discrete time parameterization.

Step 3 Estimate the new parameters for the discrete time parameterization.

Step 4 Convert this new set of parameters for discrete time parameterization back to continuous time setting using the inverse bilinear transformation.

Step 5 Convert the new parameters in compatible form to the balanced parameterization.

Step 6 Cycle through Steps 1 to 5 until convergence.

The derivation of the parameter updating algorithm is omitted in this paper due to lack of space. 

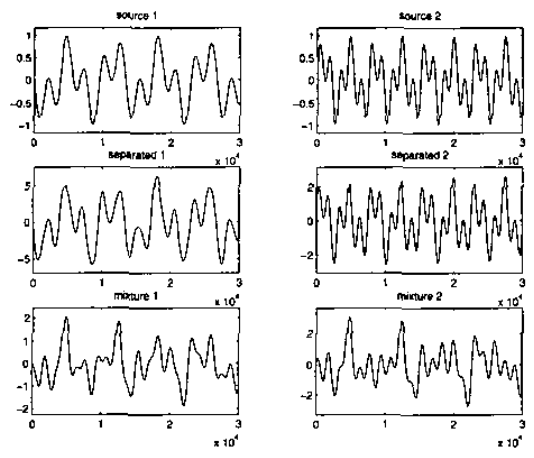

Fig. 1. The source signals, the demixed signals, and the mixed signals respectively using the balanced realization technique as proposed in this paper.
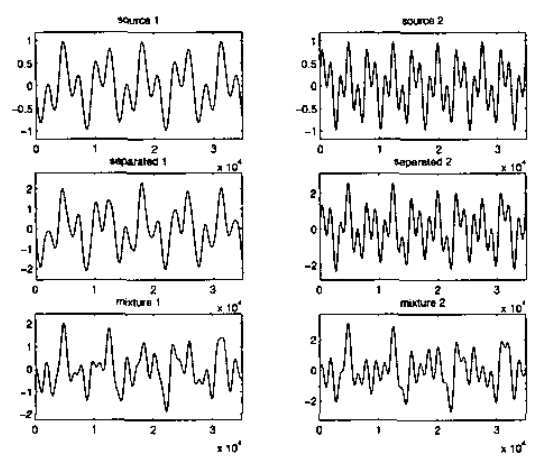

Fig. 2. The evolution of the source signals, the demixed signals, and the mixed signals using the method proposed by [8].

\section{EXPERIMENTAL RESULTS}

In order to illustrate the issues discussed in previous sections, we will consider the following experiment.

Experiment 1. $n=2, N=2$. Figure 1 shows the outputs from our proposed approach, while Figure 2 shows the output as obtained from the approach given in [8].

It is noted that in the case of evolution of the elements of matrices $D$, and $C$ the approach taken in [8] appear to result in a yet to be converged system (the parameters do not setthe into a steady state, but are oscillating around a constant value), while in the case of the balanced parameterization, the results have converged to a steady state.

In addition, though not shown here, the balanced parameterization approach does not require the number of states $N$ of the state space to be known a priori as in [8]. This is determined automatically in the process. This is an advantage over the approach taken in [8].

\section{CONCLUSION}

In this paper, we have considered the possibility of obtaining a balanced realization of the deconvolution of source signals mixed by unknown linear time invariant systems. Our method is based on the work in [8]. The contributions are threefold: this is the first time that a balanced parameterization is applied to blind deconvolution problem, secondly, in the normal balanced parameterization approach it requires access to both the input and output measurements, while in our case, we do not require the access to source signals, but instead we work only with the sensor measurements. Thirdly, we have successfully eliminated one of the assumptions in the approach by [8], viz., we do not require the number of states to be known a priori. Instead, the number of states can be obtained as part of our procedure. All we need to do is to impose an upper bound for the number of states, and then our approach will be able to estimate the number of states online.

\section{REFERENCES}

[1] S Amari, "Natural gradient works efficiently in learning", Neural Computation, Vol 10, pp 251-270, 1998.

[2] A Bell, T Sejnowski, "An information maximization approach to blind separation and blind deconvolution". Neural Computation. Vol 7, pp 1129-1159, 1995.

[3] C T Chou, Geometry of Linear Systems and Identification, PhD Thesis, Cambridge University, 1994.

[4] C T Chou, J M Maciejowski, "System Identification using Balanced Parameterization". IEEE Trans Automatic Control. Vol 42, pp 956-974, 1997.

[5] K Glover, "All optimal Hankel norm approximations of linear multivariable systems and $L^{\infty}$ error bounds". International Journal of Control. Vol 39, pp1115$1193,1984$.

[6] T Kailath, Linear Systems. Prentice Hall. 1980.

[7] R Ober, "Balanced parameterization of classes of linear systems". SIAM Journal Control and Optimization, Vol 29, pp 1251-1287, 1991.

[8] L. Zhang, A. Cichocki, "Blind Deconvolution of Dynamical Systems, A State Space Approach" Journal of Signal Processing, Vol. 4, No. 2, Mar. 2000, pp. 111130. 